\title{
Viscosity of Active Microtubules Uncovered
}

\author{
Experiments show how to tune the viscosity of "active" filaments found in \\ cells, something that could help in the design of biomimetic materials.
}

By Katherine Wright

$\square$ uring a lunchbreak several years ago, Zvonimir Dogic shared a video with his colleague Daniel Blair that captured the swirling motion of microtubules-tiny, nanoscale tubes found in cells. Blair was mesmerized by this "wild" dance and wondered aloud about the viscoelastic properties of an active microtubule-filled fluid. At the time, no one knew, but new experiments by the teams of Blair and Dogic-at Georgetown University in Washington D.C. and the University of California, Santa Barbara, respectively-have measured microtubule viscosity under a variety of conditions [1].

Microtubules form bundles when mixed in solution with the motor protein kinesin, which walks along tubules and can bind them together. These bundles fold, fray, break apart, and reform, creating an everchanging pattern of curls and coils. The rate at which these rearrangements happen, and the patterns that form, can be tuned by changing the concentration of another substance called ATP, which determines how quickly

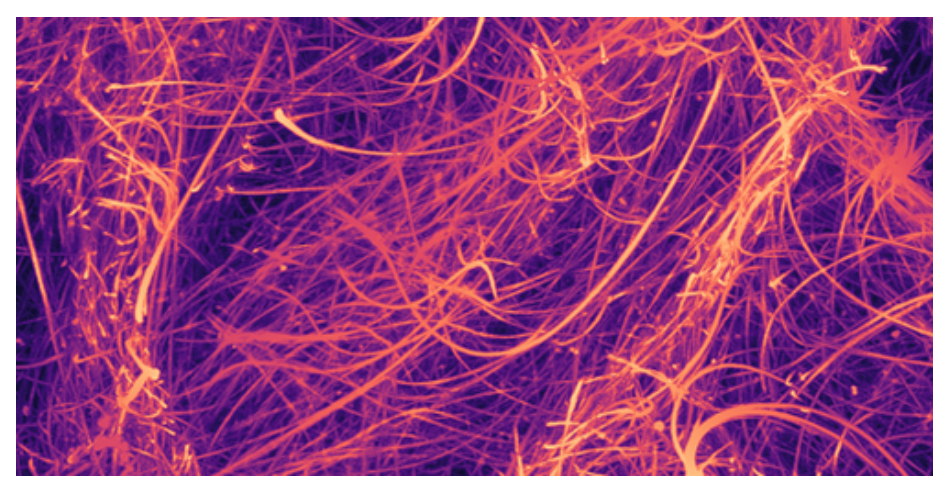

Credit: D. A. Gagnon et al. [1]

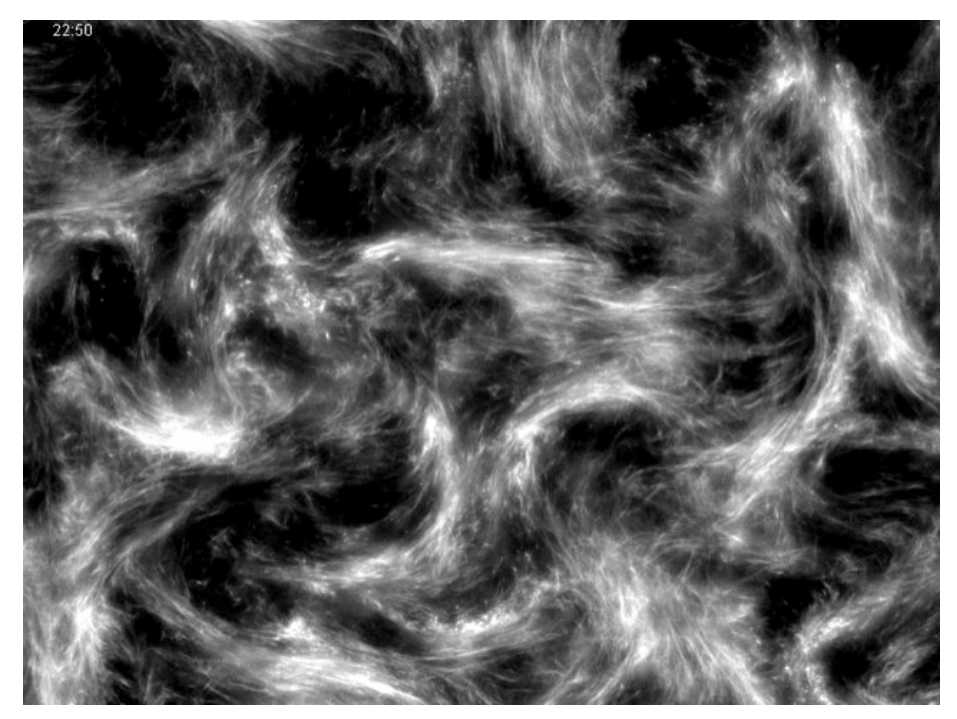

Video 1: Constantly changing patterns occur in a mixture of microtubules and kinesin.

Credit: D. Blair/Georgetown University

the kinesin walks. In their new work, Blair, Dogic, and colleagues confirm that a change in ATP concentration can also alter the mixture's rheology.

Applying a steady shear to the mixture, they found that it became more viscous (it shear-thickened) with increasing shear rate. The level of shear-thickening depended on the ATP concentration, with mixtures with a higher ATP concentration having a viscosity up to an order of magnitude lower.

Blair says that it is remarkable that the viscosity can be tuned so drastically using a single control knob (ATP concentration). He adds that this material could be used in biomimetic systems, 
such as engineered cells, the building of which will require careful control of the constituents' properties.

Katherine Wright is a Senior Editor for Physics.

\section{REFERENCES}

1. D. A. Gagnon et al., "Shear-induced gelation of self-yielding active networks," Phys. Rev. Lett. 125, 178003 (2020). 